\title{
ON THE COHOMOLOGY OF CLASSIFYING SPACES OF GROUPS OF HOMEOMORPHISMS
}

\author{
JAREK KĘDRA
}

\section{INTRODUCTION AND STATEMENT OF THE RESULTS}

Let $M$ be a closed simply connected $2 n$-dimensional manifold. The present paper is concerned with the cohomology of classifying spaces of connected groups of homeomorphisms of $M$.

1.1. Conventions. We make the following standing assumptions throughout the paper. The topology on a group of homeomorphisms of a manifold is assumed to be compact-open. We consider the cohomology with real coefficients unless otherwise specified.

\subsection{Generic coadjoint orbits.}

Theorem 1.3. Let $M=G \cdot \xi \subset \mathfrak{g}^{\vee}$ be a generic coadjoint orbit of a compact connected semisimple Lie group $G$. Suppose that the action $G \rightarrow$ $\operatorname{Homeo}(M)$ has a finite kernel. Then the homomorphism

$$
H^{*}\left(B \operatorname{Homeo}_{0}(M)\right) \rightarrow H^{*}(B G)
$$

induced by the action is surjective. It is surjective in degree four for every (not necessarily generic) coadjoint orbit.

The proof of this theorem is given in Section 5.1. Genericity means that there exists a non-empty Zariski open subset $Z \subset g^{\vee}$ of the dual of the Lie algebra of $G$ such that the theorem holds for an orbit $G \cdot \xi$, where $\xi \in Z$. We shall discuss the generic orbits in Section 6. The examples will include complex projective spaces and flag manifolds. However, the subset $Z$ for a given group $G$ is not understood.

Remark 1.4. Theorem 1.3 and most of the results in this paper can be directly generalised to a connected topological monoid of homotopy equivalences, cf. Kędra-McDuff [8].

The following result is an immediate consequence of Theorem 1.3 
Corollary 1.5. Let $M=G \cdot \xi$ be as in Theorem 1.3. Let $\mathcal{H} \subset \operatorname{Homeo}(M)$ be a connected group of homeomorphisms containing $G$ as a subgroup. Then the induced homomorphism

$$
H^{*}(B \mathcal{H}) \rightarrow H^{*}(B G)
$$

is surjective for a generic coadjoint orbit $M$ and in degree four it is surjective for all orbits.

The most important examples of groups $\mathcal{H}$ to which we apply the above result are the group $\operatorname{Ham}(M, \omega)$ of Hamiltonian diffeomorphisms, $\operatorname{Diff}_{0}(M)$ - the connected component of the identity of the group of diffeomorphism, and $\mathrm{Homeo}_{0}(M)$ - the connected component of the identity of the group of homeomorphisms.

Example 1.6. Let $M=\mathbb{C P}^{n_{1}} \times \ldots \times \mathbb{C P}^{n_{k}}$ be equipped with a product symplectic form $\omega$ invariant under the natural action of the product of special unitary groups. Then the induced homomorphism

$$
H^{*}(B \operatorname{Ham}(M, \omega)) \rightarrow H^{*}\left(B\left(\mathrm{SU}\left(n_{1}+1\right) \times \ldots \times \mathrm{SU}\left(n_{k}+1\right)\right)\right.
$$

is surjective. In particular, $\operatorname{dim} H^{4}(B \operatorname{Ham}(M, \omega)) \geq k$.

We know from [8] that the relevant characteristic classes don't vanish on spheres and hence we also have that

$$
\operatorname{rank}\left(\pi_{2 m+1}(\operatorname{Ham}(M, \omega))\right) \geq k \text { for } m \leq \min \left\{n_{1}, \ldots, n_{k}\right\} .
$$

Combining the above example (for $k=2$ ) with a theorem of Seidel [14] we immediately obtain the following result.

Theorem 1.7. Let $\mathbb{C P}^{m} \times \mathbb{C P}^{n}$ be equipped with a product symplectic form $\omega$ such that the symplectic areas of the lines in factors are equal. Suppose that $m \geq n \geq k \geq 1$. Then

$$
\operatorname{rank}\left(\pi_{2 k+1}\left(\operatorname{Diff}\left(\mathbb{C P}^{m} \times \mathbb{C P}^{n}\right)\right)\right) \geq 2 n-k+2 .
$$

1.8. Circle actions. A circle action $\mathbb{S}^{1} \rightarrow \mathcal{H} \subseteq \operatorname{Homeo}(M)$ is called $\mathcal{H}$-inessential if it defines a contractible loop in $\mathcal{H}$. For example, if a connected simply connected Lie group $G$ acts on $M$ then a circle subgroup of $G$ yields an inessential circle action. The following result generalises the second part of Theorem 1.3 . 
Theorem 1.9. Let $\left(M_{i}, \omega_{i}\right), i=1,2, \ldots, m$ be a closed simply connected symplectic manifold admitting an inessential nontrivial Hamiltonian circle action. Let $M=M_{1} \times M_{2} \times \cdots \times M_{m}$ be equipped with a product symplectic form $\omega$. If $\mathcal{H}$ is a connected group containing the product $\operatorname{Ham}\left(M_{1}, \omega_{1}\right) \times \ldots \times \operatorname{Ham}\left(M_{m}, \omega_{m}\right)$ then $\operatorname{dim} H^{4}(B \mathcal{H} ; \mathbb{R}) \geq m$ and $\operatorname{rank} \pi_{3}(\mathcal{H} ; \mathbb{R}) \geq m$.

1.10. The fibre integral subalgebra. Let $G \rightarrow \operatorname{Homeo}_{0}(M)$ be an action of a connected topological group. In Section [2, we define a certain graded subalgebra $\mathbb{A}_{G}^{*}(M) \subset H^{*}(B G)$ associated with the action. It is called the fibre integral subalgebra and it can be calculated in certain cases. It is our main technical tool and its basic properties are presented in Section 3 .

1.11. Relation to the previous work. The obvious strategy to understand the topology of the classifying space $B \mathcal{H}$ of a homeomorphism group $\mathcal{H}$ is to consider a map $f: B \rightarrow B \mathcal{H}$ defined on a space with understood topology and, for example, examine the induced map on the cohomology. In the present paper we mostly investigate the homomorphism $H^{*}(B \mathcal{H}) \rightarrow H^{*}(B G)$ for the natural action of a compact Lie group $G$ on a homogeneous space $G / H$. Conjecturally, the homomorphism

$$
H^{*}\left(B \operatorname{Homeo}_{0}(G / H)\right) \rightarrow H^{*}(B G)
$$

should be surjective for the real cohomology provided the action of $G$ is effective.

Apart from the classical results about diffeomorphisms of low dimensional spheres and surfaces the first such surjectivity result was obtained by Reznikov in [13]. He proved that the natural Hamiltonian action of $\mathrm{SU}(n)$ on the complex projective plane $\mathbb{C P}^{n-1}$ induces the surjection $H^{*}\left(B \operatorname{Ham}\left(\mathbb{C P}^{n-1}\right)\right) \rightarrow H^{*}(B \mathrm{SU}(n))$. He proved it by using a Hamiltonian version of the Chern-Weil theory. He also conjectured that a similar statement should be true for other coadjoint orbits.

The result of Reznikov was improved and generalised to flag manifolds by Kędra-McDuff in [8]. We proved that the characteristic classes defined by Reznikov are in fact topological in the sense that they can be defined in the cohomology ring of the topological monoid of homotopy equivalences of a symplectic manifold. 
The algebraic independence of the Reznikov classes was proved by Gal-Kędra-Tralle in [3] for a generic coadjoint orbit of a semisimple Lie group. It was shown by examples that his classes cannot be algebraically independent in general (see Example 6.4).

The results in [3, 8] and also [7] are applications of the fibre integral method. That is, certain characteristic classes are defined as fibre integrals. This is the main tool here as well. The new ingredient is that we consider distinct symplectic forms on a given manifold at the same time. More precisely, the Reznikov characteristic classes are equal to the fibre integrals of powers of a certain universal cohomology class called the coupling class. This class is induced by a fixed symplectic form. In this paper we consider fibre integrals of products of many coupling classes induced by distinct symplectic forms.

Acknowledgements. The present work is built upon papers [3, 8]. I thank my coauthors Dusa McDuff, Światosław Gal and Alex Tralle for discussions. I thank Dusa McDuff and Oldřich Spáčil for useful comments on a preliminary version of this paper. Any remaining mistakes are of my responsibility.

\section{A STRATEGy AND FEW TECHNICAL RESUlts}

2.1. Fibre integration. Let $M \rightarrow E \stackrel{\pi}{\rightarrow} B$ be an oriented bundle with closed $n$-dimensional fibre. There is a homomorphism of $H^{*}(B)$ modules

$$
\pi_{!}: H^{n+k}(M) \rightarrow H^{k}(B) .
$$

It is defined to be the composition

$$
H^{n+k}(E) \rightarrow E_{\infty}^{k, n} \rightarrow E_{2}^{k, n}=H^{k}\left(B ; H^{n}(M)\right)=H^{k}(B),
$$

where the $E_{m}^{p, q}$ is an $m$-th term of the associated Leray-Serre spectral sequence. The property that the fibre integration is a morphism of $H^{*}(B)$-modules means that

$$
\pi_{!}\left(\alpha \cdot \pi^{*}(\beta)\right)=\pi_{!}(\alpha) \cdot \beta .
$$

Moreover, fibre integration is multiplicative with respect to the cross product. More precisely, let $p_{1}: E_{1} \rightarrow B_{1}$ and $p_{2}: E_{2} \rightarrow B_{2}$ be oriented bundles with closed fibres. Then

$$
\left(p_{1} \times p_{2}\right) !(\alpha \times \beta)=\left(p_{1}\right) !(\alpha) \times\left(p_{2}\right) !(\beta) .
$$


This multiplicativity easily follows from the definition and good properties of the spectral sequence.

2.2. A very general view. Let $G$ be a topological group acting on a closed oriented $n$-manifold $M$. Consider the associated universal fibration

$$
M \stackrel{i}{\rightarrow} M_{G} \stackrel{\pi}{\rightarrow} B G
$$

induced by the action. Given a subalgebra $\mathbb{A} \subset H^{*}\left(M_{G}\right)$ we consider a subalgebra

$$
\left\langle\pi_{!}(\mathbb{A})\right\rangle \subset H^{*}(B G)
$$

generated by the fibre integrals of elements from $\mathbb{A}$. The strategy is to choose an appropriate subalgebra $\mathbb{A}$ for which one can make computations.

2.3. The fibre integral subalgebra. Let us assume that $M$ is simply connected and $G$ is connected and let $\mathbb{A}^{*}=\left\langle H^{2}\left(M_{G}\right)\right\rangle \subset H^{*}\left(M_{G}\right)$ be the subalgebra generated by the classes of degree 2 . Define the fibre integral subalgebra

$$
\mathbb{A}_{G}^{*}(M):=\left\langle\pi_{!}\left(\mathbb{A}^{*}\right)\right\rangle
$$

associated with the action of $G$ on $M$ to be the graded subalgebra of $H^{*}(B G)$ generated by the fibre integrals of the products of cohomology classes of degree 2. In particular, an element of $\mathbb{A}_{G}^{2 k}(M)$ is a linear combination of classes of the form $\pi_{!}\left(a_{1} \ldots a_{n+k}\right)$ where $a_{i} \in H^{2}\left(M_{G}\right)$ and $\operatorname{dim} M=2 n$. We say that the fibre integral subalgebra is full if it is equal to the whole of $H^{*}(B G)$.

2.4. The surjectivity lemma. Let $\mathcal{H} \subset \operatorname{Homeo}(M)$ be a connected group of homeomorphisms of a simply connected manifold $M$. Let

$$
M \stackrel{i}{\rightarrow} M_{\mathcal{H}} \stackrel{\pi}{\rightarrow} B \mathcal{H}
$$

be the universal bundle associated with the action of $\mathcal{H}$ on $M$.

Lemma 2.5. The homomorphism $i^{*}: H^{2}\left(M_{\mathcal{H}}\right) \rightarrow H^{2}(M)$ induced by the inclusion of the fibre is surjective.

Proof. Consider the associated Leray-Serre spectral sequence $E_{m}^{p, q}$. Since $M$ and $B \mathcal{H}$ are simply connected both the first row and the first column of the sequence are trivial. That is,

$$
E_{2}^{p, q}=H^{p}(B \mathcal{H}) \otimes H^{q}(M)=0
$$

if $p=1$ or $q=1$. 
Let $a \in H^{2}(M)=E_{2}^{0,2}$ be a nonzero cohomology class. Since $E_{2}^{2,1}=0$ we have $d_{2}(a)=0$. Thus to finish the proof we need to show that $d_{3}(a)=0$.

Since $M$ is finite dimensional there exists a number $k \in \mathbb{N}$ such that $a^{k} \neq 0$ and $a^{k+1}=0$. Observe that

$$
E_{3}^{3,2 k} \subset E_{2}^{3,2 k}=H^{3}(B \mathcal{H}) \otimes H^{2 k}(M)
$$

because the differential $d_{2}: E_{2}^{1,2 k+1} \rightarrow E_{2}^{3,2 k}$ is trivially zero, for $E_{2}^{1,2 k+1}=0$. Thus the following computation

$$
0=d_{3}\left(a^{k+1}\right)=(k+1) d_{3}(a) \otimes a^{k}
$$

implies that $d_{3}(a)=0$ are required.

Remark 2.6. The above argument proves in fact the following. Suppose that $F \rightarrow E \rightarrow B$ is a fibration over a simply connected base. Let $a \in H^{2}(F)$ be a cohomology class of finite cup-length. If $d_{2}(a)=0$ then $d_{3}(a)=0$ (cf. proof of Proposition 3.1 in [8]).

\subsection{A dimension inequality.}

Lemma 2.8. Let $M$ be a simply connected closed manifold and let $\mathcal{H} \subseteq$ Homeo $(M)$ be a connected group of homeomorphisms. Let $G \subset \mathcal{H}$ be a connected subgroup with finite $\pi_{1}(G)$. Then

$$
\operatorname{dim} \mathbb{A}_{G}^{2 k}(M) \leq \operatorname{dim} \mathbb{A}_{\mathcal{H}}^{2 k}(M) \leq \operatorname{dim} H^{2 k}(B \mathcal{H}) .
$$

In particular, if $\mathbb{A}_{G}^{*}$ is full then the homomorphism $H^{*}(B \mathcal{H}) \rightarrow H^{*}(B G)$ induced by the action is surjective.

Proof. Consider the following commutative diagram of fibrations.

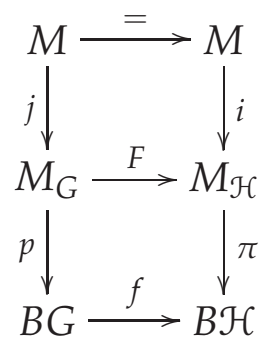

Since $\pi_{1}(G)$ is finite, $H^{2}(B G)=0$ and the inclusion of the fibre $j: M \rightarrow M_{G}$ induces an isomorphism $j^{*}: H^{2}\left(M_{G}\right) \rightarrow H^{2}(M)$. It follows from Lemma 2.5 that the homomorphism $F^{*}: H^{2}\left(M_{\mathcal{H}}\right) \rightarrow$ $H^{2}\left(M_{G}\right)$ is surjective and hence we have

$$
p_{!}\left(a_{1} \ldots a_{n+k}\right)=p_{!}\left(F^{*}\left(\tilde{a}_{1} \ldots \tilde{a}_{n+k}\right)\right)=f^{*}\left(\pi_{!}\left(\tilde{a}_{1} \ldots \tilde{a}^{n+k}\right)\right)
$$


which finishes the proof.

2.9. Cohomologically symplectic manifolds and coupling classes. A closed $2 n$-manifold $M$ is called cohomologically symplectic or shortly $c$-symplectic if there exists a class $\alpha \in H^{2}(M)$ such that $\alpha^{n} \neq 0$. Such a class $\alpha$ is called a symplectic class.

Assume that $M$ is simply connected. Let a topological group $G$ act on $M$ preserving a symplectic class $\alpha$. Let

$$
M \stackrel{i}{\rightarrow} E \stackrel{\pi}{\rightarrow} B
$$

be a fibration with the structure group $G$. There exists a unique cohomology class $\Omega_{E} \in H^{2}(E)$ such that $i^{*} \Omega_{E}=\alpha$ and $\pi_{!}\left(\Omega_{E}^{n+1}\right)=0$. The class $\Omega_{E}$ is called the coupling class. It is natural in the sense that the coupling class of a pull back bundle is the pull back of the coupling class. The symplectic class $\alpha \in H^{2}(M)$ is said to satisfy the Hard Lefschetz condition if the multiplication by its $k$-th power defines an isomorphism $H^{n-k}(M) \rightarrow H^{n+k}(M)$ for $k=0,1, \ldots, n$.

Example 2.10. All Kähler manifolds (e.g. coadjoint orbits) satisfy the Hard Lefschetz condition [4].

2.11. Consequences of the Hard Lefschetz condition. The following lemma was proved first for complex algebraic manifolds by Blanchard [1]. The proof the following topological version of the lemma can be found in Lalonde-McDuff [10].

Lemma 2.12 (Blanchard [1]). Let $M$ be a closed simply connected $c$ symplectic $2 n$-manifold satisfying the Hard Lefschetz condition. If $M \rightarrow$ $E \rightarrow B$ is a bundle with a connected structure group $\mathcal{H} \subset \operatorname{Homeo}(M)$ then the homomorphism $i^{*}: H^{*}(E) \rightarrow H^{*}(M)$ induced by the inclusion of the fibre is surjective.

Notice that the surjectivity of the homomorphism $i^{*}$ in the above lemma implies, due to the Leray-Hirsch theorem [6. Theorem 4D.1], that $H^{*}(E)$ is isomorphic as a $H^{*}(B)$-module to the tensor product $H^{*}(B) \otimes H^{*}(M)$. In particular, the homomorphism $p^{*}: H^{*}(B) \rightarrow$ $H^{*}(E)$ induced by the projection is injective.

The next proposition is motivated by the fact that if a cohomology class of a space $X$ evaluates nontrivially on a sphere then it is indecomposable. That is, it cannot be expressed as the sum of products of classes of positive degree. Hence one can think of such a class as a generator of the cohomology ring of $X$. 
Proposition 2.13. Let $M$ be a closed simply connected c-symplectic $2 n$ manifold satisfying the Hard Lefschetz conditions. Let

$$
M \stackrel{j}{\rightarrow} E \stackrel{p}{\rightarrow} S^{2 k}
$$

be a bundle over a sphere of positive dimension and with a connected structure group $\mathcal{H} \subset \operatorname{Homeo}(M)$. Let $\sigma \in H^{2 k}\left(S^{2 k}\right)$ denote a generator. If $p^{*}(\sigma)=\sum a \cdot b$, where all $a, b \in H^{*}(E)$ are of positive degree, then the homomorphism

$$
f^{*}: H^{2 k}(B \mathcal{H}) \rightarrow H^{2 k}\left(S^{2 k}\right)
$$

induced by the classifying map is surjective.

Proof. Since $M$ satisfies the Hard Lefschetz condition we have $p^{*}(\sigma) \neq 0$.

Next observe that the base sphere has to be of dimension bigger than two. Indeed, if $k=1$ then $p^{*}(\sigma)=\sum a \cdot b$ for $a, b \in H^{1}(E)$. Since $E$ is simply connected it implies that $p^{*}(\sigma)=0$, which cannot happen. Consequently we have $k>1$.

Notice that the homomorphism $j^{*}: H^{m}(E) \rightarrow H^{m}(M)$ induced by the inclusion of the fibre is an isomorphism for $m<2 k$.

Let $M \stackrel{i}{\rightarrow} M_{\mathcal{H}} \stackrel{\pi}{\rightarrow} B \mathcal{H}$ be the universal fibration and let $\Omega \in H^{2}\left(M_{\mathcal{H}}\right)$ be the coupling class associated with the symplectic class $\alpha$. Let $\hat{a}, \hat{b} \in H^{*}\left(M_{\mathcal{H}}\right)$ be such that $i^{*} \hat{a}=j^{*} a$ and $i^{*} \hat{b}=j^{*} b$.

In the following calculation, $\Omega_{E}=F^{*}(\Omega)$ denotes the coupling class. Also, since $j^{*}$ is an isomorphism in degrees smaller than $2 k$, we have $F^{*}(\hat{a})=a$ and $F^{*}(\hat{b})=b$. This implies the second equality.

$$
\begin{aligned}
f^{*} \pi_{!}\left(\Omega^{n} \cdot \sum \hat{a} \cdot \hat{b}\right) & =p_{!}\left(F^{*}\left(\Omega^{n} \cdot \sum \hat{a} \cdot \hat{b}\right)\right) \\
& =p_{!}\left(\Omega_{E}^{n} \cdot \sum a \cdot b\right) \\
& =p_{!}\left(\Omega_{E}^{n} \cdot p^{*} \sigma\right)=\sigma \cdot \operatorname{volume}(M)
\end{aligned}
$$

\section{BASIC PROPERTIES OF THE FIBRE INTEGRAL SUBALGEBRA}

Throughout this section $M$ and $N$ are assumed to be closed, connected and simply connected manifolds. 
Proposition 3.1. Let $H \rightarrow G \rightarrow \operatorname{Homeo}_{0}(M)$ be a sequence of actions of connected topological groups on a manifold $M$. Let $f: B H \rightarrow B G$ denote the induced map. If $f^{*}: H^{2}(B G) \rightarrow H^{2}(B H)$ is surjective then

$$
\mathbb{A}_{H}^{*}(M) \subset f^{*}\left(\mathbb{A}_{G}^{*}(M)\right) \text {. }
$$

Proof. Consider the diagram of universal fibrations.

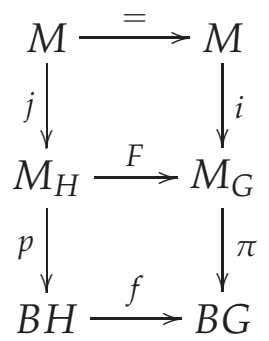

Let $p_{!}\left(\Omega_{1} \ldots \Omega_{k}\right) \in \mathbb{A}_{H}^{*}(M)$, where $j^{*}\left(\Omega_{i}\right)=a_{i}$. According to Lemma 2.5, there are classes $\widehat{\Omega}_{i} \in H^{2}\left(M_{G}\right)$ such that $i^{*}\left(\widehat{\Omega}_{i}\right)=a_{i}$. Since $H$ and $G$ are connected their classifying spaces are simply connected and we have

$$
\Omega_{i}-F^{*}\left(\widehat{\Omega}_{i}\right)=p^{*}\left(\alpha_{i}\right)
$$

for some $\alpha_{i} \in H^{2}(B H)$. It follows from the hypothesis that $\alpha_{i}=$ $f^{*}\left(\beta_{i}\right)$ and we get

$$
\Omega_{i}=F^{*}\left(\widehat{\Omega}_{i}-\pi^{*}\left(\beta_{i}\right)\right)
$$

We finally have

$$
p_{!}\left(\Omega_{1} \ldots \Omega_{k}\right)=f^{*} \pi_{!}\left(\left(\widehat{\Omega}_{1}-\pi^{*} \beta_{1}\right) \ldots\left(\widehat{\Omega}_{k}-\pi^{*} \beta_{k}\right)\right)
$$

which finishes the proof.

Proposition 3.2. Let $G$ and $H$ be connected groups acting on manifolds $M$ and $N$ respectively. Then $G \times H$ acts on $M \times N$ and

$$
\mathbb{A}_{G \times H}^{*}(M \times N) \cong \mathbb{A}_{G}^{*}(M) \otimes \mathbb{A}_{H}^{*}(N) \text {. }
$$

In particular, if both $\mathbb{A}_{G}^{*}(M)$ and $\mathbb{A}_{H}^{*}(N)$ are full then $\mathbb{A}_{G \times H}^{*}(M \times N)$ is also full.

Proof. The statement is true due to the multiplicativity property of the fibre integration with respect to the cross product and the isomorphism $H^{*}(B H \times B G)=H^{*}(B H) \otimes H^{*}(B G)$. 
Proposition 3.3. Let a connected group $G$ act on $M$ and $N$. The cup product in $H^{*}(B G)$ induces a map

$$
\mathbb{A}_{G}^{*}(M) \otimes \mathbb{A}_{G}^{*}(N) \rightarrow H^{*}(B G)
$$

with the image equal to $\mathbb{A}_{G}^{*}(M \times N)$. In particular, if $G$ acts on $N$ trivially then $\mathbb{A}_{G}^{*}(M)=\mathbb{A}_{G}^{*}(M \times N)$.

Proof. The map in the statement is the composition

$$
\mathbb{A}_{G}^{*}(M) \otimes \mathbb{A}_{G}^{*}(N) \stackrel{\cong}{\longrightarrow} \mathbb{A}_{G \times G}^{*}(M \times N) \stackrel{\Delta^{*}}{\longrightarrow} H^{*}(B G)
$$

where the first isomorphism is due to Proposition 3.2 and the second map is induced by the diagonal $\triangle: B G \rightarrow B G \times B G$.

It follows from Proposition 3.1 that $\mathbb{A}_{G}^{*}(M \times N)$ is contained in the image of the above map. Thus we need to show that the converse inclusion holds,

$$
\Delta^{*}\left(\mathbb{A}_{G \times G}^{*}(M \times N)\right) \subset \mathbb{A}_{G}^{*}(M \times N) .
$$

According to simple connectivity we have that

$$
H^{2}\left(M_{G} \times N_{G}\right)=H^{2}\left(M_{G}\right) \oplus H^{2}\left(N_{G}\right)
$$

and hence an element in the subalgebra of $H^{*}\left(M_{G} \times N_{G}\right)$ generated by degree two classes is a sum of products of the form $\left(\alpha_{1} \ldots \alpha_{k}\right) \times$ $\left(\beta_{1} \ldots \beta_{l}\right)$, for $\alpha_{i} \in H^{2}\left(M_{G}\right)$ and $\beta_{i} \in H^{2}\left(N_{G}\right)$. Since this is itself a product of degree two classes we get that its pull back via the map $\widehat{\Delta}:(M \times N)_{G} \rightarrow M_{G} \times N_{G}$ is a product of degree two classes. This, according to the functoriality of the fibre integration, finishes the proof.

Lemma 3.4. Suppose that $G$ is a connected group with finite $\pi_{1}(G)$ acting on a closed simply connected $2 n$-manifold $M$. Then the fibre integral subalgebra $\mathbb{A}_{G}^{*}(M)$ is generated by the fibre integrals of powers, i.e. by the classes of the form $\pi_{!}\left(a^{m}\right)$.

Proof. Let $A \in H_{2 k}(B G)$ be a homology class. A certain nonzero multiple of $A$ is represented by a map $f: B \rightarrow B G$ defined on a closed oriented connected $2 k$-manifold $B$. Suppose that

$$
f^{*}\left(\pi_{!}\left(a_{1} \ldots a_{n+k}\right)\right) \neq 0,
$$

where $a_{i} \in H^{2}\left(M_{G}\right)$. The map $f$ induces a bundle $M \rightarrow E \rightarrow B$ and the above inequality is equivalent to

$$
0 \neq F^{*}\left(a_{1} \ldots a_{n+k}\right) \in H^{2(n+k)}(E)=\mathbb{R},
$$


where $F: E \rightarrow M_{G}$ is the induced map of total spaces. It follows that the product map

$$
F^{*}\left(H^{2}\left(M_{\mathcal{H}}\right)\right) \otimes \ldots \otimes F^{*}\left(H^{2}\left(M_{\mathcal{H}}\right)\right) \rightarrow H^{2(n+k)}(E)=\mathbb{R}
$$

is nontrivial. Since it is a polynomial map, due to the polarisation formula, the power map $F^{*}(a) \mapsto F^{*}(a)^{n+k}$ is also nontrivial.

This shows that $f^{*}\left(\pi_{!}\left(a^{n+k}\right)\right) \neq 0$ for some class $a \in H^{2}\left(M_{G}\right)$.

We have shown that for every homology class in $A \in H_{2 k}(B G)$ which evaluates nontrivially on the fibre integral subalgebra there exists a class $a \in H^{2}\left(M_{G}\right)$ such that the fibre integral of its $(n+k)$ th power evaluates nontrivially on $A$. This proves that such fibre integrals generate $\mathbb{A}_{G}^{*}(M)$.

Remark 3.5. Since the power map $F^{*}\left(H^{2}\left(M_{\mathcal{H}}\right)\right) \rightarrow H^{n+k}(E)$ in the above proof is polynomial and nontrivial, there exists a nonempty open and dense subset $U \subset F^{*}\left(H^{2}\left(M_{\mathcal{H}}\right)\right)$ such that $a^{n+k} \neq 0$ for $a \in U$.

\section{Calculations for coadjoint orbits}

4.1. Symplectic preliminaries. Let $G$ be a compact connected semisimple Lie group and let $\xi \in \mathfrak{g}^{\vee}$ be a covector. The coadjoint orbit $G \cdot \xi$ admits a G-invariant symplectic form. The Killing form provides an equivariant isomorphism between the Lie algebra $\mathfrak{g}$ and its dual $\mathfrak{g}^{\vee}$ and hence also a bijective correspondence between adjoint and coadjoint orbits.

Let $T \subset G$ be a maximal torus and denote by $\mathfrak{t}$ its Lie algebra. Every adjoint orbit has a representative in $t$. The Lie algebra $t$ is decomposed into the Weyl chambers. Let $C \subset \mathfrak{t}$ denote the closure of a Weyl chamber. It is a polyhedral cone. If two elements $\xi, \eta \in \mathfrak{t}$ belong to the interior of a face of $C$ then the corresponding adjoint orbits $G \cdot \xi$ and $G \cdot \eta$ are diffeomorphic. In this case the isotropy groups $G_{\xi}$ and $G_{\eta}$ are conjugate in $G$. The conjugation by an element of $G$ provides a $G$-equivariant diffeomorphism between the orbits $G \cdot \xi$ and $G \cdot \eta$.

Thus we can fix one orbit $M$ and consider it as a smooth manifold equipped with various $G$-invariant symplectic forms. Since the conjugation induces a map of $B G$ homotopic to the identity the universal fibration $M \rightarrow M_{G} \rightarrow B G$ is Hamiltonian with respect to these symplectic forms. In such a case we have the coupling class 
$\Omega_{\xi} \in H^{2}\left(M_{G}\right)$ corresponding to the symplectic form on the orbit $G \cdot \xi \cong M$.

4.2. Flag manifolds. Let $\operatorname{dim} G / T=2 n$ and consider the universal bundle $G / T \rightarrow B T \stackrel{\pi}{\rightarrow} B G$ associated with the action.

Lemma 4.3. The fibre integral subalgebra $\mathbb{A}_{G}^{*}(G / T)$ is full. Moreover, it is generated by the fibre integrals of powers of coupling classes.

Proof. Let $\operatorname{dim} G / T=2 n$ and let $G / T \rightarrow B T \stackrel{\pi}{\rightarrow} B G$ be the universal bundle associated with the action. Consider the following composition

$$
H^{2}(B T) \otimes \cdots \otimes H^{2}(B T) \rightarrow H^{2(n+k)}(B T) \stackrel{\pi_{1}}{\rightarrow} H^{2 k}(B G),
$$

where the first map is the product and the second is the fibre integration. Observe the first map is polynomial and surjective since $H^{*}(B T)$ is the polynomial algebra generated by $H^{2}(B T)$. The second map is surjective which follows from the injectivity of $\pi^{*}: H^{*}(B G) \rightarrow$ $H^{*}(B T)$. Indeed, if $b \in H^{2 k}(B G)$ then $\pi_{!}\left(\pi^{*}(b) \cdot \Omega^{n}\right)=b$, for a coupling class $\Omega \in H^{2}(B T)$. This proves that the fibre integral subalgebra is full.

The second statement follows from Lemma 3.4 and Remark 3.5. Indeed, there exists an open and dense subset of $H^{2}(B T)$ consisting of coupling classes.

4.4. Fibre integral of a power of the coupling class as an invariant polynomial. The cohomology of the classifying space of a compact Lie group is isomorphic to the algebra of invariant polynomials on the Lie algebra

$$
H^{2 k}(B G) \cong S^{k}\left(\mathfrak{g}^{\vee}\right)^{G} .
$$

The latter is isomorphic to $S^{k}(\mathfrak{t})^{W_{G}}$, the polynomials on the Lie algebra of the maximal torus invariant under the Weyl group of $G$. The next lemma follows from [8, Lemma 3.6 and Lemma 3.9].

Lemma 4.5. Let $M=G \cdot \xi$ be a $2 n$-dimensional coadjoint orbit of a semisimple Lie group $G$. The fibre integral of the $(n+k)$-th power of the coupling class $\Omega_{\xi} \in H^{2}\left(M_{G}\right)$ corresponds to the following invariant polynomial.

$$
P_{k}(\xi, X):=(-1)^{k}\left(\begin{array}{c}
n+k \\
k
\end{array}\right) \cdot \int_{G}\left\langle X, \operatorname{Ad}_{g}^{\vee}(\xi)\right\rangle^{k} \operatorname{vol}_{G}
$$


Since the polynomials $P_{k}(\xi, X)$ depend continuously (with respect to the Zariski topology) on $\xi$ and since the algebraic independence is an open condition we obtain the following result.

Proposition 4.6. Let $\xi \in \mathfrak{g}^{\vee}$. There exists a Zariski open neighbourhood $Z \subset \mathfrak{g}^{\vee}$ of $\xi$ such that

$$
\mathbb{A}_{G}^{*}(G \cdot \xi) \subset \mathbb{A}_{G}^{*}(G \cdot \eta)
$$

for every $\eta \in Z$.

Corollary 4.7. If $M=G \cdot \xi \subset \mathfrak{g}^{\vee}$ is a generic coadjoint orbit of a compact semisimple Lie group $G$ then the fibre integral subalgebra $\mathbb{A}_{G}^{*}(M)$ is full.

Proof. It follows from Proposition 4.6 that if there is an orbit with the full fibre integral subalgebra then a generic coadjoint orbit has full fibre integral subalgebra. The statement follows from Lemma 4.3.

\section{Proofs of Theorem 1.3 and Theorem 1.9}

5.1. Proof of Theorem 1.3. According to Corollary 4.7 we have that the fibre integral subalgebra $\mathbb{A}_{G}^{*}(M)$ is full for a generic coadjoint orbit $M$. Then, since $G$ is semisimple its fundamental group is finite, the first statement follows from Lemma 2.8 .

To prove the second statement observe first that a compact semisimple group $G$ is finitely covered by a product $G_{1} \times \ldots \times G_{m}$ of simple groups. Thus there is a splitting of the Lie algebra $\mathfrak{g}=\mathfrak{g}_{1} \oplus \ldots \oplus \mathfrak{g}_{m}$.

The composition $G_{1} \times \ldots \times G_{m} \rightarrow G \rightarrow \operatorname{Aut}\left(\mathfrak{g}^{\vee}\right)$ of the covering projection and the coadjoint action is the coadjoint action of the product and hence it is the product of coadjoint actions $\mathrm{Ad}^{\vee}: G_{i} \rightarrow$ $\operatorname{Aut}\left(\mathfrak{g}_{i}^{\vee}\right)$. Thus $M$ is diffeomorphic to a product $M_{1} \times \ldots \times M_{m}$ of the corresponding coadjoint orbits of simple groups.

Next we want to show that each of the above orbits $M_{i}$ is of positive dimension. This follows from the assumption on the kernel of the action. Indeed, since the kernel of the action $\operatorname{Ad}^{\vee}: G \rightarrow \operatorname{Aut}\left(\mathfrak{g}^{\vee}\right)$ is finite, so is the kernel of the coadjoint action of the product. But the latter is isomorphic to the product of the kernels of $\mathrm{Ad}^{\vee}: G_{i} \rightarrow$ $\operatorname{Aut}\left(\mathfrak{g}^{\vee}\right)$ and hence each $M_{i}$ is a nontrivial orbit.

The statement now follows from Theorem 1.9 proof of which we shall present next. 
5.2. Proof of Theorem 1.9, Let $\operatorname{dim} M=2 n, \operatorname{dim} M_{i}=2 n_{i}$ and let $\omega_{a}=\sum a_{i} \omega_{i}$, where $a:=\left(a_{1}, \ldots a_{m}\right)$ is an $m$-tuple of positive real numbers. Let $\Omega_{a} \in H^{2}\left(M_{\mathcal{H}}\right)$ be the coupling class associated with the symplectic form $\omega_{a}$.

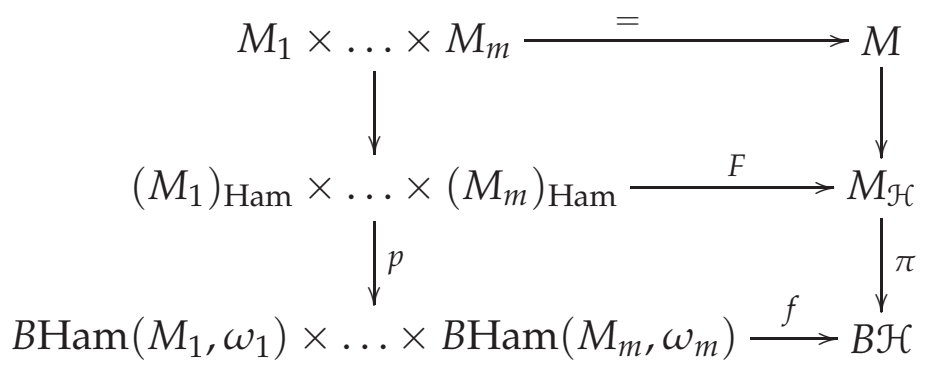

The pull-back of the coupling class $F^{*}\left(\Omega_{a}\right)$ is equal to the sum of coupling classes $\sum a_{i} \Omega_{i}$. In the following computation $C_{k}$ is a positive constant depending on a tuple $k=\left(k_{1}, \ldots, k_{m}\right)$ of non-negative integers and the fourth equality follows from the multiplicativity of the fibre integration with respect to the cross product.

$$
\begin{aligned}
f^{*}\left(\pi_{!}\left(\Omega_{a}\right)^{n+2}\right) & =p_{!}\left(F^{*}\left(\Omega_{a}\right)^{n+2}\right) \\
& =p_{!}\left(\left(\sum a_{i} \Omega_{i}\right)^{n+2}\right) \\
& =\sum C_{k} \cdot p_{!}\left(\left(a_{1} \Omega_{1}\right)^{k_{1}} \times \ldots \times\left(a_{m} \Omega_{m}\right)^{k_{m}}\right) \\
& \left.=\sum C_{k} \cdot\left(p_{1}\right)_{!}\left(\left(a_{1} \Omega_{1}\right)^{k_{1}}\right) \times \ldots \times\left(p_{m}\right)_{!}\left(a_{m} \Omega_{m}\right)^{k_{m}}\right)
\end{aligned}
$$

Notice that the fibre integral $\left(p_{i}\right)_{!}\left(\Omega_{i}^{k}\right)=0$ if $k<n_{i}$. Thus the above sum is, up to a positive constant, equal to the sum of terms of the form

$$
\left(p_{1}\right) !\left(a_{1} \Omega_{1}\right)^{n_{1}} \times \ldots \times\left(p_{i}\right) !\left(a_{i} \Omega_{i}\right)^{n_{i}+2} \times \ldots \times\left(p_{m}\right)_{!}\left(a_{m} \Omega_{m}\right)^{n_{m}} .
$$

It follows from [8, Theorem 1.1] that an inessential nontrivial circle action induces an element of $\sigma_{i} \in \pi_{4}\left(B \operatorname{Ham}\left(M_{i}\right)\right)$ on which the class $\left(p_{i}\right) !\left(\Omega_{i}^{n+2}\right)$ evaluates nontrivially. By varying the $m$-tuple $a=$ $\left(a_{1}, \ldots, a_{m}\right)$ we obtain that the image of the homomorphism

$$
f^{*}: H^{4}(B \mathcal{H}) \rightarrow H^{4}\left(B \operatorname{Ham}\left(M_{1}, \omega_{1}\right) \times \ldots \times B \operatorname{Ham}\left(M_{m}, \omega_{m}\right)\right)
$$

is at least $m$-dimensional.

Choosing the parameters $a$ appropriately, the classes $\pi_{!}\left(\Omega_{a}^{n+2}\right)$ define $m$ linearly independent functionals on $\pi_{4}(B \mathcal{H}) \otimes \mathbb{R}$. Evaluating 
them on the images of the classes $\sigma_{i}$ we obtain that the rank $\pi_{4}(B \mathcal{H})$ is at least $m$. This rank is equal to the rank of $\pi_{3}(\mathcal{H})$.

\section{EXAMPLES}

Let $G$ be a compact connected semisimple Lie group with a maximal torus $T$. Let $\mathfrak{g}$ and $\mathfrak{t}$ denote the corresponding Lie algebras. The closed positive Weyl chamber $C \subset \mathfrak{t}$ is a simplicial cone. Let $F$ be a face of $C$. If $\xi$ and $\eta$ belong to the interior of $F$ then they are diffeomorphic. Moreover, if $\xi \in$ interior $(F)$ then

$$
\operatorname{dim} H^{2}(G \cdot \xi)=\operatorname{dim} F .
$$

Conversely, a coadjoint orbit $G \cdot \xi$ has a representative that belongs to the interior of a face of dimension equal to $\operatorname{dim} H^{2}(G \cdot \xi)$.

Remark 6.1. The above observations can be easily deduced from Bott's results in [2] (see also Section 2 of his survey in [11] and Section 2.3 of Guillemin-Lerman-Sternberg [5]).

Proposition 4.6 states that $\mathbb{A}_{G}^{*}(G \cdot \xi) \subset \mathbb{A}_{G}^{*}(G \cdot \eta)$ for any $\eta$ in an open and dense neighbourhood $U \subset C$ of $\xi$. Thus the most interesting are the orbits corresponding to the edges (i.e. one-dimensional faces) of $C$. This is because near an edge there are points corresponding to many topologically distinct orbits. The orbits corresponding to edges are characterised by their second Betti number being equal to one. They are called minimal in the terminology of Guillemin-Lerman-Sternberg [5].

Example 6.2. The minimal coadjoint orbits of $S U(n)$ are the complex grassmannians $\mathrm{G}(k, n)$.

6.3. Special unitary group $S U(n)$. It is known that the fibre integral subalgebra associated with the natural projective action of $\mathrm{SU}(n)$ on the complex projective space $\mathbb{C P}^{n-1}$ is full [8, Proposition 1.7]. Thus it follows from Proposition 4.6 that every coadjoint orbit $M$ of $\mathrm{SU}(n)$ close to the projective space has full fibre integral subalgebra $\mathbb{A}_{\mathrm{SU}(n)}^{*}(M)$. Such orbits are of the form

$$
\mathrm{SU}(n) / \mathrm{S}\left(\mathrm{U}\left(n_{1}\right) \oplus \ldots \oplus \mathrm{U}\left(n_{k}\right) \oplus \mathrm{U}(1)\right)
$$

where $n_{1}+\ldots+n_{k}+1=n$. 
Example 6.4 (Proposition 3.6 in [3]). The fibre integral subalgebra of the complex grassmannian $\mathrm{G}(n, 2 n)$ with respect to the natural action of $\mathrm{SU}(2 n)$ is not full. More precisely, $H^{6}(B \mathrm{SU}(2 n))$ is not contained in the fibre integral subalgebra. This is due to the fact that the relevant invariant polynomial has odd degree and its zero represents the grassmannian (see [3, Section 3] for more examples and details).

Remark 6.5. It is shown in [8, Proposition 4.8] that the natural action of $\mathrm{SU}(n)$ on a generalised flag manifold $M$ induces a surjective homomorphism $H^{*}\left(B \mathrm{Homeo}_{0}(M)\right) \rightarrow H^{*}(B \mathrm{SU}(n))$. The proof is also an application of fibre integrals. It is, however, specialised to this particular case.

6.6. Special orthogonal group $\mathrm{SO}(2 m), m>2$. The cohomology ring of the classifying space of the special orthogonal group is generated by the Pontryagin classes $p_{1}, \ldots, p_{m}$ and the Euler class $e$. They have degrees $\operatorname{deg}\left(p_{k}\right)=4 k$ and $\operatorname{deg}(e)=2 m$.

Consider a minimal coadjoint orbit of the form $M:=\mathrm{SO}(2 m) / \mathrm{U}(m)$. The classes $p_{1}, e, p_{m+1}, \ldots, p_{2 m}$ belong to the fibre integral subalgebra $\mathbb{A}_{\mathrm{SO}(2 m)}^{*}(M)$. For the first Pontryagin class this follows from the second part of Theorem 1.3 .

To see that the the higher Pontryagin classes $p_{k}$ for $k>m$ belong to the fibre integral subalgebra consider the map $\pi: B \mathrm{U}(m) \rightarrow$ $B \mathrm{SO}(2 \mathrm{~m})$ induced by the inclusion. The pullback of a Pontryagin class is expressed in terms of Chern classes by the well-known formula

$$
\pi^{*}\left(p_{k}\right)=c_{k}^{2}-2 c_{k-1} c_{k+1}+\ldots \pm 2 c_{2 k} .
$$

Thus if $k>m$ then $\pi^{*}\left(p_{k}\right)$ is a sum of products of classes of positive degrees and Proposition 2.13 applies.

Finally, a result of Reznikov [9, 13] implies that the Euler class belongs to the fibre integral subalgebra.

Since $H^{2}(M)=\mathbb{R}$ there are, in general, orbits close to $M$ that are topologically different from $M$. Their fibre integral subalgebras contain $\mathbb{A}_{\mathrm{SO}(2 m)}^{*}(M)$.

Remark 6.7. We excluded the case of $\mathrm{SO}(4)$ because then the action of the group $\mathrm{SO}(4)$ on the orbit $\mathrm{SO}(4) / \mathrm{U}(2)=\mathbb{C P}^{1}$ is not effective. 


\section{The FIBRe INTEgRAL SUbALGEBRA IS STABLE}

If $G$ acts on a manifold $M$ then it also acts on the product $M \times N$ (acting trivially on the second factor). We think of the composition of actions

$$
f: G \rightarrow \operatorname{Homeo}(M) \rightarrow \operatorname{Homeo}(M \times N)
$$

as a kind of stabilisation. If $G$ is connected then the second part of Proposition 3.3 states that $\mathbb{A}_{G}^{*}(M)=\mathbb{A}_{G}^{*}(M \times N)$ and hence

$$
\mathbb{A}_{G}^{*}(M) \subset f^{*}\left(B \operatorname{Homeo}_{0}(M \times N)\right) .
$$

This means that the part of the topology of the classifying space of the group of homeomorphisms of $M$ captured by the fibre integral subalgebra persists when we stabilise $M$.

Example 7.1. Let $M$ be a coadjoint orbit of a semisimple compact Lie group $G$. Let $N$ be a closed simply connected symplectic manifold. Then the fibre integral subalgebra $\mathbb{A}_{G}^{*}(M)$ is contained in the image of the homomorphism

$$
H^{*}(B \operatorname{Ham}(M \times N)) \rightarrow H^{*}(B G)
$$

induced by the action of $G$ on the product. In particular if the fibre integral subalgebra is full then the above homomorphism is surjective.

Remark 7.2. The fundamental group of the group of Hamiltonian diffeomorphisms of a product symplectic manifold has been recently investigated by Pedroza in [12].

\section{REFERENCES}

[1] André Blanchard. Sur les variétés analytiques complexes. Ann. Sci. Ecole Norm. Sup. (3), 73:157-202, 1956.

[2] Raoul Bott. An application of the Morse theory to the topology of Lie-groups. Bull. Soc. Math. France, 84:251-281, 1956.

[3] Światosław R. Gal, Jarek Kędra, and Aleksy Tralle. On algebraic independence of hamiltonian characteristic classes. Journal of Symplectic Geometry, to appear, arXiv:1005.2038.

[4] Phillip Griffiths and Joseph Harris. Principles of algebraic geometry. Wiley Classics Library. John Wiley \& Sons Inc., New York, 1994. Reprint of the 1978 original.

[5] Victor Guillemin, Eugene Lerman, and Shlomo Sternberg. Symplectic fibrations and multiplicity diagrams. Cambridge University Press, Cambridge, 1996.

[6] Allen Hatcher. Algebraic topology. Cambridge University Press, Cambridge, 2002. 
[7] Tadeusz Januszkiewicz and Jarek Kędra. Characteristic classes of smooth fibrations. math.SG/0209288.

[8] Jarek Kędra and Dusa McDuff. Homotopy properties of hamiltonian group actions. Geom. Topol., 9:121-162 (electronic), 2005.

[9] Jarek Kędra, Aleksy Tralle, and Artur Woike. On nondegenerate coupling forms. Journal of Geometry and Physics, to appear, arXiv:1004.3699.

[10] François Lalonde and Dusa McDuff. Symplectic structures on fiber bundles. Topology, 42(2):309-347, 2003.

[11] G. L. Luke, editor. Representation theory of Lie groups, volume 34 of London Mathematical Society Lecture Note Series, Cambridge, 1979. Cambridge University Press.

[12] Andrés Pedroza. Seidel's representation on the Hamiltonian group of a Cartesian product. Int. Math. Res. Not. IMRN, (14):Art. ID rnno49, 19, 2008.

[13] Alexander G. Reznikov. Characteristic classes in symplectic topology. Selecta Math. (N.S.), 3(4):601-642, 1997. Appendix D by Ludmil Katzarkov.

[14] Paul Seidel. On the group of symplectic automorphisms of $\mathrm{CP}^{m} \times \mathrm{CP}^{n}$. In Northern California Symplectic Geometry Seminar, volume 196 of Amer. Math. Soc. Transl. Ser. 2, pages 237-250. Amer. Math. Soc., Providence, RI, 1999.

UNIVERSITY OF AbERDEEN AND UNIVERSITY OF SZCZECIN

E-mail address: kedra@abdn.ac.uk 